\title{
Formation of compact galaxies in the Extreme-Horizon simulation
}

\author{
S. Chabanier ${ }^{1,2}$, F. Bournaud ${ }^{2,1}$, Y. Dubois ${ }^{3}$, S. Codis ${ }^{3,4}$, D. Chapon ${ }^{1}$, D. Elbaz ${ }^{2}$, C. Pichon ${ }^{3,4,5}$, O. Bressand ${ }^{6,7}$, \\ J. Devriendt ${ }^{8}$, R. Gavazzi ${ }^{3}$, K. Kraljic ${ }^{9}$, T. Kimm ${ }^{10}$, C. Laigle ${ }^{3}$, J.-B. Lekien ${ }^{6,7}$, G. Martin ${ }^{11,12}$, \\ N. Palanque-Delabrouille ${ }^{1}$, S. Peirani ${ }^{13}$, P.-F. Piserchia ${ }^{6,7}$, A. Slyz ${ }^{14}$, M. Trebitsch ${ }^{15,16}$, and C. Yèche ${ }^{1}$ \\ ${ }^{1}$ IRFU, CEA, Université Paris-Saclay, 91191 Gif-sur-Yvette, France \\ e-mail: chabaniersolene@gmail.com \\ 2 AIM, CEA, CNRS, Université Paris-Saclay, Université Paris Diderot, Sorbonne Paris Cité, 91191 Gif-sur-Yvette, France \\ 3 CNRS and Sorbonne Université, UMR 7095, Institut d'Astrophysique de Paris, 98 bis Boulevard Arago, 75014 Paris, France \\ ${ }^{4}$ Université Paris-Saclay, CNRS, CEA, Institut de Physique Théorique, 91191 Gif-sur-Yvette, France \\ 5 Korea Institute of Advanced Studies (KIAS) 85 Hoegiro, Dongdaemun-gu, Seoul 02455, Republic of Korea \\ 6 CEA, DAM, DIF, 91297 Arpajon, France \\ 7 Université Paris-Saclay, CEA, Laboratoire en Informatique Haute Performance pour le Calcul et la simulation, \\ 91680 Bruyères-le-Châtel, France \\ 8 Astrophysics, University of Oxford, Oxford OX1 3RH, UK \\ ${ }^{9}$ Institute for Astronomy, Royal Observatory, Edinburgh EH9 3HJ, UK \\ 10 Department of Astronomy, Yonsei University, 50 Yonsei-ro, Seodaemun-gu, Seoul 03722, Republic of Korea \\ 11 Steward Observatory, University of Arizona, 933 N. Cherry Ave, Tucson, AZ 85719, USA \\ 12 Korea Astronomy and Space Science Institute, 776 Daedeokdae-ro, Yuseong-gu, Daejeon 34055, Korea \\ 13 Université Côte d'Azur, Observatoire de la Côte d'Azur, CNRS, Laboratoire Lagrange, CS 34229, 06304 Nice Cedex 4, France \\ 14 Astrophysics, University of Oxford, Denys Wilkinson Building, Keble Road, Oxford OX1 3RH, UK \\ 15 Max-Planck-Institut für Astronomie, Königstuhl 17, 69117 Heidelberg, Germany \\ 16 Zentrum für Astronomie der Universität Heidelberg, Institut für Theoretische Astrophysik, Albert-Ueberle-Str. 2, \\ 69120 Heidelberg, Germany
}

Received 9 June 2020 / Accepted 3 October 2020

\begin{abstract}
We present the Extreme-Horizon (EH) cosmological simulation, which models galaxy formation with stellar and active galactic nuclei (AGN) feedback and uses a very high resolution in the intergalactic and circumgalactic medium. Its high resolution in low-density regions results in smaller-size massive galaxies at a redshift of $z=2$, which is in better agreement with observations compared to other simulations. We achieve this result thanks to the improved modeling of cold gas flows accreting onto galaxies. In addition, the EH simulation forms a population of particularly compact galaxies with stellar masses of $10^{10-11} M_{\odot}$ that are reminiscent of observed ultracompact galaxies at $z \simeq 2$. These objects form primarily through repeated major mergers of low-mass progenitors and independently of baryonic feedback mechanisms. This formation process can be missed in simulations with insufficient resolution in low-density intergalactic regions.
\end{abstract}

Key words. galaxies: formation - galaxies: evolution - galaxies: high-redshift - galaxies: structure - methods: numerical

\section{Introduction}

Early-type galaxies (ETGs) at redshifts of $z>1.5$ are much more compact than nearby ones (Daddi et al. 2005). At stellar masses about $10^{11} M_{\odot}$, they typically have half-mass radii of $0.7-3 \mathrm{kpc}$, which is about three times smaller than nearby ellipticals with similar masses (van der Wel et al. 2014). Compact radii are typically accompanied by steep luminosity profiles and high Sersic indices (van Dokkum \& Brammer 2010; Carollo et al. 2013). Star-forming galaxies (SFGs) also decrease in size with increasing redshift (e.g., Kriek et al. 2009; Dutton et al. 2011). In addition, the CANDELS survey has discovered a population of very compact SFGs at $z \simeq 2$, that is, so-called "blue nuggets" (Barro et al. 2013; Williams et al. 2014), which have stellar masses of $10^{10-11} M_{\odot}$ and unusually small effective radii around $2 \mathrm{kpc}$ and sometimes even below $1 \mathrm{kpc}$. Compact SFGs have high comoving densities, of about $10^{-4} \mathrm{Mpc}^{-3}$ for stellar masses above $10^{10} M_{\odot}$ and $10^{-5} \mathrm{Mpc}^{-3}$ above $10^{11} M_{\odot}$ (Wang et al. 2019). Also, SFGs at $z \simeq 2$ often have very compact gas and star formation distributions (Elbaz et al. 2018).

A number of processes have been proposed to explain the formation of compact galaxies, ranging from early formation in a compact Universe (Lilly \& Carollo 2016) and the compaction of initially extended galaxies (Zolotov et al. 2015) and up through processes that may include galaxy mergers, disk instabilities (Bournaud et al. 2007a; Dekel \& Burkert 2014), triaxial haloes (Tomassetti et al. 2016), accretion of counter-rotating gas (Danovich et al. 2015), and gas return from a low-angular momentum fountain (Elmegreen et al. 2014).

The Extreme-Horizon (EH) cosmological simulation, presented in Sect. 2, models galaxy-formation processes with the same approach as Horizon-AGN (HAGN, Dubois et al. 2014, 
Table 1. Resolution strategy for EH and SH.

\begin{tabular}{ccccccccc}
\hline \hline Comoving grid resolution $\left[\mathrm{kpc} \mathrm{h}^{-1}\right]$ & 97.6 & 48.8 & 24.4 & 12.2 & 6.1 & 3.05 & 1.52 & 0.76 \\
Physical grid resolution $[\mathrm{kpc}](z=2)$ & 47 & 23.5 & 11.7 & 5.8 & 2.9 & 1.5 & 0.7 & 0.3 \\
\hline Volume fraction $(\mathrm{EH})(z=2)$ & - & $45 \%$ & $43 \%$ & $10 \%$ & $1 \%$ & $0.04 \%$ & $z<2$ & $z<2$ \\
Volume fraction $(\mathrm{SH})(z=2)$ & $80 \%$ & $17 \%$ & $2 \%$ & $0.17 \%$ & $0.013 \%$ & $5 \times 10^{-4} \%$ & $z<2$ & $z<2$ \\
Volume fraction $(\mathrm{HAGN})(z=2)$ & $77 \%$ & $19 \%$ & $2 \%$ & $0.2 \%$ & $0.01 \%$ & $6 \times 10^{-4} \%$ & $z<2$ & $z<2$ \\
\hline
\end{tabular}

Notes. The first two lines indicate the comoving and physical $(\mathrm{at} z=2)$ grid resolution in $\mathrm{kpc} \mathrm{h}^{-1}$ and $\mathrm{kpc}$, respectively. The last three lines indicate the volume fractions measured at each resolution level at $z=2$ in EH, SH and HAGN for comparison. In the last two columns, $z<2$ means that these levels are not triggered yet at $z=2$ but will be for lower redshifts.

hereafter D14) and with a substantially increased resolution in the intergalactic and circumgalactic medium (IGM and CGM). The properties of massive galaxies in $\mathrm{EH}$ and the origin of their compactness are discussed in Sects. 3 and 4.

\section{The Extreme-Horizon simulation}

The EH simulation is performed with the adaptive mesh refinement code RAMSES (Teyssier 2002) using the physical models from HAGN (D14). The spatial resolution in the CGM and IGM is largely increased compared to HAGN, while the resolution inside galaxies is identical, at the expense of a smaller box size of $50 \mathrm{Mpc} \mathrm{h}^{-1}$. The control simulation of the same box with a resolution similar to HAGN is called Standard-Horizon (SH). EH and $\mathrm{SH}$ share initial conditions realized with mpgrafic (Prunet et al. 2008). These use a $\Lambda$ CDM cosmology with matter density $\Omega_{\mathrm{m}}=0.272$, dark energy density $\Omega_{\Lambda}=0.728$, matter power spectrum amplitude $\sigma_{8}=0.81$, baryon density $\Omega_{\mathrm{b}}=0.0455$, Hubble constant $\mathrm{H}_{0}=70.4 \mathrm{~km} \mathrm{~s}^{-1} \mathrm{Mpc}^{-1}$, and scalar spectral index $n_{\mathrm{s}}=0.967$, based on the WMAP-7 cosmology (Komatsu et al. 2011). The EH simulation was performed on 25000 cores of the AMD-Rome partition of the Joliot Curie supercomputer at TGCC and it partly used the Hercule parallel I/O library (Bressand et al. 2012; Strafella \& Chapon 2020). It is being run down to $z \sim 0$.

\subsection{Resolution strategy}

The SH simulation uses a $512^{3}$ coarse grid, with a minimal resolution of $100 \mathrm{kpch}^{-1}$ as in HAGN. Cells are refined up to a resolution of $\simeq 1 \mathrm{kpc}$ in a quasi-Lagrangian manner: any cell is refined if $\rho_{\mathrm{DM}} \Delta x^{3}+\left(\Omega_{\mathrm{b}} / \Omega_{\mathrm{DM}}\right) \rho_{\text {baryon }} \Delta x^{3}>m_{\text {refine,SH }} M_{\mathrm{DM}, \mathrm{res}}$, where $\rho_{\text {DM }}$ and $\rho_{\text {baryon }}$ are dark matter (DM) and baryon densities respectively in the cell, $\Delta x^{3}$ is the cell volume, and $m_{\text {refine,SH }}=$ 80 . The $\Omega_{\mathrm{b}} / \Omega_{\mathrm{DM}}$ factor ensures that baryons dominate the refinement condition as soon as there is a baryon overdensity. This resolution strategy matches that of HAGN (Table 1).

The EH simulation uses a $1024^{3}$ coarse grid and a more aggressive refinement strategy with $m_{\text {refine,EH }}=1 / 40 m_{\text {refine,SH }}$ in the IGM/CGM (for $\Delta x>1.52 \mathrm{kpc} \cdot \mathrm{h}^{-1}$ ), but with $m_{\text {refine, } \mathrm{EH}}=$ $m_{\text {refine,SH }}$ near to and in galaxies: the whole volume is resolved with a resolution that is twice as high and most of the mass is resolved with a four times higher resolution in $1 \mathrm{D}$, yielding an improvement of 8 to 64 for the 3-D resolution. This improvement continues until the highest resolution of $\simeq 1 \mathrm{kpc}$ is reached; the volume fraction at various resolution levels are listed in Table 1. Such an aggressive approach for grid refinement can better model the early collapse of structures (O'Shea et al. 2005). Appendix A illustrates the resolution achieved in representative regions of the CGM and IGM in $\mathrm{EH}$ and $\mathrm{SH}$. The resolution in $\mathrm{EH}$ haloes is typically $\sim 6 \mathrm{kpc}$, while it is $\sim 25 \mathrm{kpc}$ for $\mathrm{SH}$.
However, galaxies themselves are treated at the very same resolution in EH and SH: any gas denser than $0.1 \mathrm{~cm}^{-3}$ is resolved at the highest level in $\mathrm{SH}$, as is also the case for $90 \%$ of the stellar mass.

\subsection{Baryonic physics}

Like in HAGN (D14), reionization takes place after a redshift of 10 due to heating from a uniform UV background from Haardt \& Madau (1996). There is $\mathrm{H}$ and $\mathrm{He}$ cooling implemented as well as metal cooling, following the Sutherland \& Dopita (1993) model.

Star formation occurs in cells with a hydrogen number density larger than $\rho_{0}=0.1 \mathrm{H} / \mathrm{cm}^{3}$. The star formation rate density is $\dot{\rho}_{*}=\epsilon_{*} \rho / t_{\mathrm{ff}}$, where $t_{\mathrm{ff}}$ is the local gas free-fall time and $\epsilon_{*}=0.02$ is the star-formation efficiency (Kennicutt 1998). Mass, energy, and metals are released by stellar winds, with type Ia and type II supernovae, assuming a Salpeter Initial Mass Function.

Black holes $(\mathrm{BH})$ are represented by sink particles with an initial mass of $10^{5} M_{\odot}$. They accrete gas through an Eddington-limited Bondi-Hoyle-Lyttleton model. Boosted accretion episodes are included when the gas density overcomes a density threshold aimed at mitigating resolution effects, with the boosting calibrated to produce realistic $\mathrm{BH}$ masses. The AGN feedback comes in two modes (Dubois et al. 2012): the quasar mode injects thermal energy and the radio mode injects mass, momentum, and kinetic energy into the surrounding medium. For a detailed parameterization of these models, we refer to D14, the analysis of Dubois et al. (2016), and Dubois et al. (2012).

\section{Galaxy compaction in EH}

\subsection{Galaxies in the $\mathrm{EH}$ simulation}

We detected galaxies with more than 50 stellar particles (about $10^{8} M_{\odot}$ ) using AdaptaHOP (Aubert et al. 2004). There are 37698 galaxies detected in EH at $z \sim 2$ and 20314 in SH, with stellar mass functions at various redshifts shown in Fig. 1. While the mass functions above $10^{10} M_{\odot}$ are quite similar in both simulations, EH forms twice as many galaxies as $\mathrm{SH}$ with stellar $M_{*} \leq 5 \times 10^{9} M_{\odot}$. We rule out any detection bias since stellar particles have similar masses in $\mathrm{EH}$ and $\mathrm{SH}$ (new stars form at the maximal resolution level in each simulation) and we attribute this difference to the increased resolution in low-density regions. Fitting the $z=2$ mass function with a power law of the form $\Phi\left(M_{*}\right) \propto M_{*}^{\beta}$ in the $10^{9} \leq \log \left(M_{*} / M_{\odot}\right) \leq 10^{9.5}$ range yields $\beta=-0.68$ for $\mathrm{EH}$ and -0.34 for $\mathrm{SH}$. Observations indicate a slope of $-1.0 \leq \beta \leq-0.5$ in this mass range (Santini et al. 2012; Tomczak et al. 2014), demonstrating that low-mass galaxy formation is substantially under-resolved or delayed in SH. 

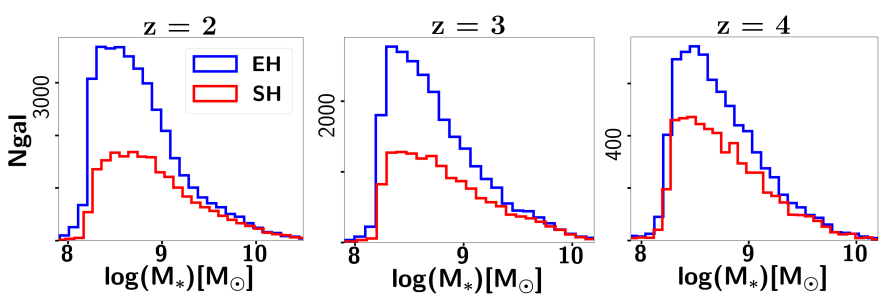

Fig. 1. Number of galaxies per mass bin in $\mathrm{EH}$ and $\mathrm{SH}$ at $z=2,3$, and 4.

We built samples of galaxies with $M_{*} \geq 5 \times 10^{10} M_{\odot}$. Ongoing major mergers identified through the presence of a companion with more than $20 \%$ of the stellar mass within $20 \mathrm{kpc}$ or a double nucleus were rejected, yielding a sample of massive galaxies (displayed in Appendix B for each simulation). We then studied the mass distribution of the selected galaxies, taking into account their non-sphericity. Stellar density maps were computed with a $500 \mathrm{pc}$ pixel size. Pixels below $50 M_{\odot} \mathrm{pc}^{-2}$, typically corresponding to a surface brightness $\mu_{i} \geq$ $28 \mathrm{mag} \mathrm{arcsec}^{-2}$, are blanked out. Ellipse-fitting of iso-density contours is performed using the technique from Krajnović et al. (2006) taking into account the $500 \mathrm{pc}$ pixel size and the $\sim 1 \mathrm{kpc}$ PSF.

Satellite galaxies are removed according to the following process: the circular region centered on the luminosity peak of the companion and extending up to the saddle of the luminosity profile between the main galaxy and the companion is ignored in the ellipse-fitting procedure and replaced with the density profile modeled on other regions. Satellites with a mass below $5 \%$ of the main galaxy are ignored to avoid removing sub-structures of the main galaxy. Three perpendicular projections are analyzed for each galaxy and the median results are kept for both the stellar mass $M_{*}$ and the half-mass radius $R_{e}$, with the latter defined as the semi-major axis of the isophote-fitting ellipse containing $50 \%$ of the stellar mass. The removal of satellite galaxies and low-density outskirts yields final stellar masses slightly below the initial estimates, down to $M_{*} \simeq 3-4 \times 10^{10} M_{\odot}$.

Stellar masses and radii are shown at $z=2$ in Fig. 2. Here, $83 \%$ of the galaxies in our sample are on the main sequence of star formation (MS; Elbaz et al. 2011), so that we can compare their size to the model from Dutton et al. (2011), which is known to provide a good fit to MS galaxies at $z=2^{1}$. The SH galaxies are larger than both the EH galaxies and observed MS galaxies. The EH galaxies generally lie around the observed relation and a small fraction of them have significantly smaller sizes. We define the compactness, $C$, as the ratio between the radius expected from the Dutton et al. (2011) model and the actual radius. The compactness distribution for $\mathrm{EH}$ (Fig. 3) peaks at around $C \simeq 1$ but it exhibits a distinct tail for $C>1.3$. We thus define two massive galaxy populations in EH: 10 ultra-compact (UC) galaxies with $C>1.3$ and 50 non ultra-compact (NUC) ones.

In summary, massive galaxies in $\mathrm{EH}$ are globally more compact than in $\mathrm{SH}$ and $\mathrm{EH}$ contains a population of UC outliers. The larger sizes in SH do not just correspond to extended stellar haloes: the difference remains when we vary the surface density threshold in mock images and Sersic indices are, on average, similar in both EH and SH. The size difference is not expected to arise from internal processes such as instabilities or feedback given that galactic scales and feedback are treated with the

\footnotetext{
1 In the mass range studied here, the Dutton et al. model lies between the mass-size relations derived at $z \simeq 1.75$ and $z \simeq 2.25$ for SFGs in CANDELS by van der Wel et al. (2014).
}

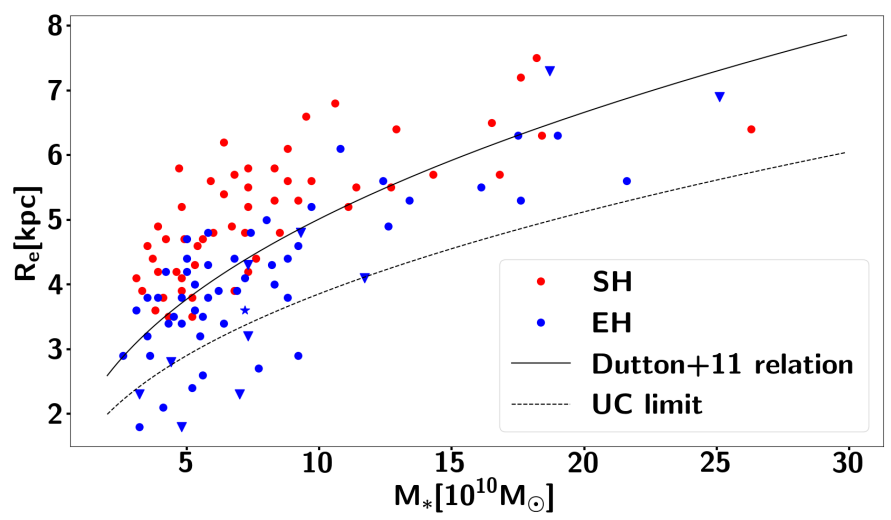

Fig. 2. Stellar half-mass radius $R_{e}$ versus stellar mass $M_{*}$ for massive galaxies at $z=2$ in $\mathrm{EH}$ and $\mathrm{SH}$. The displayed model from Dutton et al. (2011) provides a good fit to SFGs at $z=2$. UC galaxies lie below the black dashed line while NUC galaxies are above. We identify EH galaxies above and below the Main Sequence of star formation (MS) with stars and triangles, respectively, following the definition of the MS from Schreiber et al. (2017).

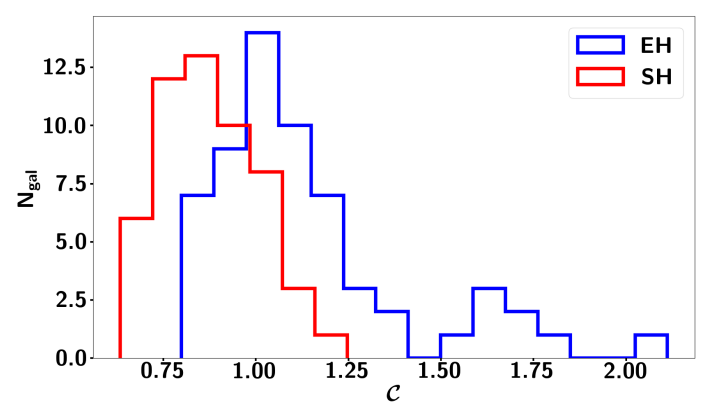

Fig. 3. Compactness distributions for the $\mathrm{EH}$ and $\mathrm{SH}$ massive galaxies at $z=2$.

very same resolution in $\mathrm{EH}$ and $\mathrm{SH}$. Two key differences could contribute to these effects: EH models gas flows in the CGM at a much higher resolution and low-mass galaxies are underresolved in $\mathrm{SH}$.

\subsection{Diffuse accretion and angular momentum supply}

A substantial part of the angular momentum of galaxies is supplied by cold gas inflows (Ocvirk et al. 2008; Pichon et al. 2011; Danovich et al. 2015; Tillson et al. 2015), which are better resolved in EH. Higher resolution could also better probe metal mixing in the IGM and subsequent cooling (Scannapieco et al. 2006). To probe these potential effects, we focus on inflowing gas in the vicinity of massive galaxies using the following criteria, which typically select inflowing gas according to other simulations (e.g., Goerdt et al. 2015): (1) a galactocentric radius between $3 R_{e}$ and $50 \mathrm{kpc}$; (2) a density below $0.1 \mathrm{~cm}^{-3}$ to exclude satellites; (3) a velocity vector pointing inwards w.r.t. the galaxy center; (4) a temperature below $10^{5.5} \mathrm{~K}$.

For each resolution element following these criteria, we compute the gas mass, $m$, and angular momentum, $l$, with regard to the galaxy center (in norm, $l=\|l\|$ ), then sum up the total angular momentum, $L=\Sigma l$, and mass, $M=\Sigma m$, for inflowing gas, and compute the angular momentum of inflowing gas $\mathcal{L}_{\text {in }}=L / M$ around each galaxy. Differences in $\mathcal{L}_{\text {in }}$ for various galaxy samples are listed in Table 2, demonstrating that $\mathcal{L}_{\text {in }}$ around massive galaxies is substantially lower in $\mathrm{EH}$ than in $\mathrm{SH}$, 
Table 2. Mean difference in the specific angular momentum of inflowing gas $\mathcal{L}_{\text {in }}$ between several samples of massive galaxies.

\begin{tabular}{lc}
\hline \hline Galaxy samples & Mean difference in $\mathcal{L}_{\text {in }}$ \\
\hline EH vs. SH & $13 \%$ lower \\
EH-NUC vs. SH & $10 \%$ lower \\
EH vs. SH at $M_{*}<10^{11} M_{\odot}$ & $12 \%$ lower \\
EH-UC vs. EH-NUC at $M_{*}<10^{11} M_{\odot}$ & $3 \%$ lower \\
\hline
\end{tabular}

but it is rather similar around EH-UC and EH-NUC galaxies. Taking the magnitude of the vector sum instead yields results in agreement to within a few percent and leads to the same general conclusion $^{2}$.

We can estimate the potential impact on galaxy sizes under two extreme assumptions. On the one hand, if the circular velocity remains unchanged, dominated by a non-contracting DM halo, then the galactic radii should follow $R \propto \mathcal{L}_{\text {in }}$. On the other hand, if the dark matter halo contracts in the same proportions as the baryons, the rotation velocity $V$ and radius $R$ follow $V^{2} \propto 1 / R$ at fixed mass, such that $R \propto \mathcal{L}_{\text {in }}^{2}$.

As a result, the $10 \%$ difference in $\mathcal{L}_{\text {in }}$ between EH-NUC and SH could lead to a 10-20\% difference in size: this could account for the smaller sizes of massive galaxies in $\mathrm{EH}$ as compared to $\mathrm{SH}$. On the other hand, the population of UC galaxies does not result from diffuse gas accretion as it only has the capacity to impact sizes by a few percent as compared to NUC galaxies.

Angular momentum is built up by tidal torques that only depend on very large-scale structures that are expected to be well-resolved even in SH (Fall \& Efstathiou 1980). However, angular momentum can be lost when cold inflowing streams interact with hot gas haloes and outflows in the CGM. Idealized simulations of cold streams interacting with hot haloes (Mandelker et al. 2020a,b) indicate that instabilities can decrease the velocity of cold streams by up to a few tens of percent in favorable cases, which can explain the loss of angular momentum at the $\mathrm{EH}$ resolution compared to $\mathrm{SH}^{3}$.

\subsection{Major mergers of low-mass progenitors}

Another driver of compaction could be the numerous low-mass galaxies in $\mathrm{EH}$ that are missing in $\mathrm{SH}$. We identify the progenitors of $z=2 \mathrm{UC}$ and NUC galaxies by tracking their stellar particles, and analyze their progenitors at $z=3$ and $z=4$ with the same technique as in our $z=2$ sample.

Figure 4 shows the compactness as a function of the mass ratios between each $z=2$ galaxy and its main $z=3$ progenitor and between the main $z=3$ progenitor and the second and third most massive progenitors. UC galaxies have (1) a main $z=3$ progenitor that never exceeds $10 \%$ of the $z=2$ mass, along with the (2) second and (3) third most massive progenitors that are almost as massive as the main progenitor, with mass ratios lower than 3:1 (generally lower than 2:1) for the second most massive, and generally below $4: 1$ for the third most massive. This points directly to a correlation between these param-

\footnotetext{
2 The differences in angular momentum of inflowing gas listed in Table 2 are marginally larger when considering the magnitude of the vector sum.

3 Mandelker et al. suggest that 10-20 resolution elements per stream diameter are required to model such instabilities. For our typical filament diameter of $20-30 \mathrm{kpc}$ at $z=2-3$, EH reaches such resolution in the CGM, but SH does not (Appendix A, Fig. A.2).
}

eters, showing that the formation of EH-UC galaxies involves repeated ${ }^{4}$ major mergers between low-mass progenitors. These mergers occur rapidly between $z=3$ and $z=2$, with $80 \%$ of UC galaxies assembling $90 \%$ of their stellar mass in this redshift range. Conversely, $70 \%$ of galaxies that have assembled $90 \%$ of their stellar mass between $z=3$ and $z=2$ end up as UC galaxies.

In contrast, EH-NUC and $\mathrm{SH}$ galaxies most often have one dominant progenitor undergoing only minor mergers and very rarely meet the three criteria depicted above for UC formation at the same time. There is actually no SH galaxy and only one EH-NUC galaxy that lies in the three shaded areas in Fig. 4 at the same time. This strengthens our argument that these specific types of accretion histories essentially always produce UC galaxies. The only exception among EH-NUC galaxies has an extended spiral disk morphology and has the second highest total angular momentum $L$ in inflowing gas over the whole EH sample so that accretion of diffuse gas compensates for the compacting effects of the merger history in this extreme object. This is expected based on idealized simulations of repeated mergers with various mass ratios that see merger histories involving mostly major mergers with relatively similar masses producing more concentrated end products for the same total merged mass (at least in terms of Sersic indices, Bournaud et al. 2007b, Fig. 4). Furthermore, $45 \%$ and $47 \%$ of the stars found in EHNUC and SH galaxies at $z=2$ are already formed at $z=3$, respectively, compared to only $36 \%$ for EH-UC galaxies: UC galaxies arise from low-mass progenitors and hence form their stars later on.

We also note that the distributions of progenitor masses are fairly identical for EH-NUC and SH galaxies (Fig. 4), indicating that the smaller sizes of EH-NUC galaxies do not result from different merger histories but, rather, from the modeling of the diffuse gas infall (Sect. 3.3).

\section{Discussion}

In order to match the resolution of SH and HAGN in galaxies, the EH simulation is limited to kpc-scale resolution, so the real compactness of UC galaxies could be under-estimated given they are as compact as the resolution limit allows. Zoom-in simulations will be required to make robust assessment of their size distribution. Nevertheless, the population of UC galaxies in EH is tightly associated with specific formation histories that are dominated by major mergers of low-mass progenitors, as compared to larger galaxies in the simulation.

To further probe the effect of feedback in compact galaxy formation, we used the Horizon-AGN suite of simulations from Chabanier et al. (2020). These simulations are run with extreme feedback parameters, leading to insufficiently realistic variations of the black hole-to-stellar mass ratio, yet the average galaxy size at fixed stellar mass changes by less than $10 \%$, confirming that feedback is not a key driver of the formation of UC galaxies in $\mathrm{EH}$.

Thus far, we have analyzed the compactness of galaxies independently from their star-formation activity. As expected for galaxies in the $10^{10}-10^{11} M_{\odot}$ stellar mass range at $z=2$, both NUC and UC galaxies are mostly star-forming galaxies on the MS. There is, nevertheless, a clear trend for compact galaxies to have relatively low specific star formation rates (sSFR, Fig. 5). The majority of UC galaxies lie on the low-sSFR end of the MS, as has been observed for blue nuggets (Barro et al. 2017).

\footnotetext{
4 Similar criteria hold for the fourth and fifth most massive progenitors and are also valid when the same analysis is performed at $z=4$.
} 


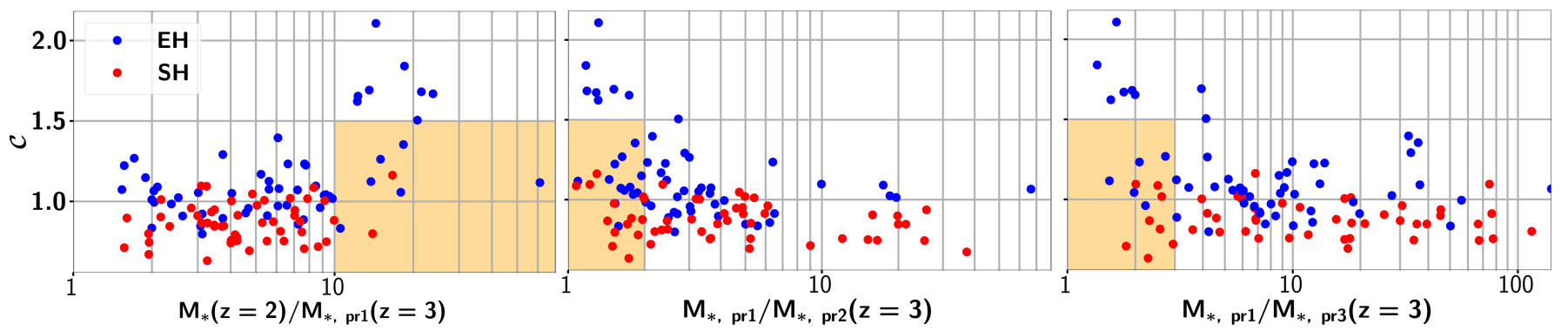

Fig. 4. Compactness, $C$, as a function of the ratio between the stellar mass at $z=2, M_{*}(z=2)$, and the mass of the main progenitor at $z=3$ $M_{*, \operatorname{pr} 1}(z=3)$ (left panel) and as a function of the ratios between the mass of the three most massive progenitors (first and second with $M_{*}$ pr2 on the middle panel; first and third with $M_{*}$, pr3, on the right panel) for EH (blue) and SH (red) galaxies. The shaded areas define regions of galaxies that would grow through major mergers of low-mass galaxies but would end up as NUC: there is no SH galaxy and only one EH galaxy in the three areas at the same time.

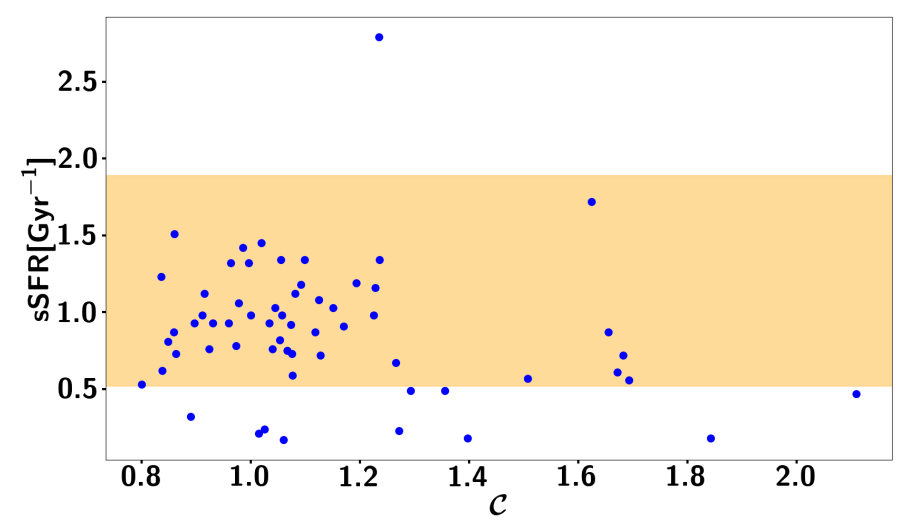

Fig. 5. Specific star formation rate (sSFR) as a function of compactness $(C)$ for EH galaxies at $z=2$. The shaded area defines the Main Sequence following Schreiber et al. (2017).

The relatively low sSFRs of UCs, as well as the tentative excess of galaxies below the MS among UCs as compared to NUCs, are consistent with the idea that these objects are undergoing quenching through gas exhaustion, feedback, or a combination of both (Tacchella et al. 2016).

The number of UC galaxies in $\mathrm{EH}(10$ objects in $\left.\left(50 \mathrm{Mpch}^{-1}\right)^{3}\right)$ is consistent with the number density of compact SFGs (see Introduction). The EH volume is too small to firmly probe the formation of massive compact ETGs at $z=2$ given that statistically, just about one such object is expected in this volume, but the excess of low-mass progenitors in EH is already present at $z=4$ (Fig. 1) and this could explain the early formation of such compact ETGs. There is indeed one galaxy in EH with $M_{*}=1.2 \times 10^{11} M_{\odot}$ and compactness $C=1.29$ (almost UC in our definition), with a low sSFR $=0.23 \mathrm{Gyr}^{-1}$ (a factor 7 below the MS), a low gas fraction of $11 \%$ (within $3 R_{e}$ ), and a Sersic index of 3.6 at $z=2$. This galaxy continues to quench into a compact ETG by redshift $z \simeq 1.8$, with $\mathrm{sSFR}=0.13 \mathrm{Gyr}^{-1}, M_{*}=1.7 \times 10^{11} M_{\odot}$, and $R_{e}=4.0 \mathrm{kpc}$ at $z \simeq 1.8$, thus lying close to the mass-size relation of ETGs at $z=1.75$ from van der Wel et al. (2014). This candidate compact ETG also forms through major mergers of low-mass progenitors: its two main progenitors at $z=4$ contain $11 \%$ and $8 \%$ of its stellar mass, respectively.

We also examined the environment of UC and NUC galaxies in $\mathrm{EH}$ by studying the large-scale structure with the persistent skeleton approach (Sousbie 2011); UC galaxies are found in relatively dense environments, but not in the very densest filaments and nodes (see Appendix C). This strengthens our previous findings on the merger history of UC galaxies, as objects in the densest regions of the main filaments are expected to form their main progenitor early on and subsequently grow through minor mergers and diffuse accretion.

\section{Conclusion}

In this Letter, we introduce the $\mathrm{EH}$ cosmological hydrodynamical simulation that is based on the physical model of HAGN, with a substantial increase in the spatial resolution in the IGM and CGM and with galactic scales treated at the same resolution. The SH simulation of the same volume uses a lower resolution in the CGM and IGM, which is more typical in cosmological simulations.

The comparison of the mass-size relation of massive galaxies in EH and SH highlights the importance of modeling diffuse gas flows at a high-enough resolution in the IGM and CGM, as this tends to reduce the angular momentum supply onto massive galaxies. In addition, the EH simulation produces a population of ultracompact (UC) galaxies. These form rapidly through repeated major mergers of low-mass progenitors, which can be missed in simulations using a modest resolution in low-density regions. One pleasing outcome of our analysis is that issues in galaxy formation simulations could indeed be solved by accurately resolving structure formation without calling upon feedback or novel subgrid models.

Acknowledgements. The Extreme-Horizon simulation was performed as a "Grand Challenge" project granted by GENCI on the AMD Rome extension of the Joliot Curie supercomputer at TGCC. We are indebted to Marc Joos, Adrien Cotte, Christine Ménaché and the whole HPC Application Team at TGCC for their efficient support. Collaborations and discussions with Bruno Thooris, Eric Armengaud, Marta Volonteri, Avishai Dekel, are warmly acknowledged. We deeply appreciate comments from Jérémy Blaizot on the Extreme Horizon project. This research used the RAMSES code written mainly by Romain Teyssier, the custom HERCULE parallel I/O library, the KINEMETRY package written by Davor Krajnović, and the DISPERSE code from Thierry Sousbie. This work was supported by the ANR 3DGasFlows (ANR-17-CE31-0017) and made use of the Horizon Cluster hosted by the Institut d'Astrophysique de Paris, run by Stéphane Rouberol. SCo's research is partially supported by Fondation MERAC. TK was supported by the National Research Foundation of Korea (NRF-2017R1A5A1070354 and NRF-2020R1C1C100707911). MT is supported by Deutsche Forschungsgemeinschaft (DFG, German Research Foundation) under Germany's Excellence Strategy EXC-2181/1 - 390900948 (the Heidelberg STRUCTURES Cluster of Excellence).

\section{References}

Aubert, D., Pichon, C., \& Colombi, S. 2004, MNRAS, 352, 376 
Barro, G., Faber, S. M., Pérez-González, P. G., et al. 2013, ApJ, 765, 104 Barro, G., Faber, S. M., Koo, D. C., et al. 2017, ApJ, 840, 47

Bournaud, F., Elmegreen, B. G., \& Elmegreen, D. M. 2007a, ApJ, 670, 237 Bournaud, F., Jog, C. J., \& Combes, F. 2007b, A\&A, 476, 1179

Bressand, O., Colombet, L., Fontaine, A., Harel, G., \& Lekien, J.B. 2012, CHOCS, 41, 29 (http://www-physique-chimie.cea.fr/ science-en-ligne/docs/chocs/Chocs_41.pdf)

Carollo, C. M., Bschorr, T. J., Renzini, A., et al. 2013, ApJ, 773, 112

Chabanier, S., Bournaud, F., \& Dubois, Y. 2020, MNRAS, 495, 1825

Daddi, E., Renzini, A., Pirzkal, N., et al. 2005, ApJ, 626, 680

Danovich, M., Dekel, A., Hahn, O., Ceverino, D., \& Primack, J. 2015, MNRAS, 449, 2087

Dekel, A., \& Burkert, A. 2014, MNRAS, 438, 1870

Dubois, Y., Devriendt, J., Slyz, A., \& Teyssier, R. 2012, MNRAS, 420, 2662

Dubois, Y., Pichon, C., Welker, C., et al. 2014, MNRAS, 444, 1453

Dubois, Y., Peirani, S., Pichon, C., et al. 2016, MNRAS, 463, 3948

Dutton, A. A., van den Bosch, F. C., Faber, S. M., et al. 2011, MNRAS, 410 , 1660

Elbaz, D., Dickinson, M., Hwang, H. S., et al. 2011, A\&A, 533, A119

Elbaz, D., Leiton, R., Nagar, N., et al. 2018, A\&A, 616, A110

Elmegreen, B. G., Struck, C., \& Hunter, D. A. 2014, ApJ, 796, 110

Fall, S. M., \& Efstathiou, G. 1980, MNRAS, 193, 189

Goerdt, T., Ceverino, D., Dekel, A., \& Teyssier, R. 2015, MNRAS, 454, 637

Haardt, F., \& Madau, P. 1996, ApJ, 461, 20

Kennicutt, R. C., Jr. 1998, ApJ, 498, 541

Komatsu, E., Smith, K. M., Dunkley, J., et al. 2011, ApJS, 192, 18

Krajnović, D., Cappellari, M., de Zeeuw, P. T., \& Copin, Y. 2006, MNRAS, 366, 787
Kriek, M., van Dokkum, P. G., Franx, M., Illingworth, G. D., \& Magee, D. K. 2009, ApJ, 705, L71

Lilly, S. J., \& Carollo, C. M. 2016, ApJ, 833, 1

Mandelker, N., Nagai, D., Aung, H., et al. 2020a, MNRAS, 494, 2641

Mandelker, N., van den Bosch, F. C., Nagai, D., et al. 2020b, MNRAS, 498, 2415

Ocvirk, P., Pichon, C., \& Teyssier, R. 2008, MNRAS, 390, 1326

O'Shea, B. W., Nagamine, K., Springel, V., Hernquist, L., \& Norman, M. L. 2005, ApJS, 160, 1

Pichon, C., Pogosyan, D., Kimm, T., et al. 2011, MNRAS, 418, 2493

Prunet, S., Pichon, C., Aubert, D., et al. 2008, ApJS, 178, 179

Santini, P., Fontana, A., Grazian, A., et al. 2012, A\&A, 538, A33

Scannapieco, E., Pichon, C., Aracil, B., et al. 2006, MNRAS, 365, 615

Schreiber, C., Pannella, M., Leiton, R., et al. 2017, A\&A, 599, A134

Sousbie, T. 2011, MNRAS, 414, 350

Sousbie, T. 2013, ArXiv e-prints [arXiv:1302.6221]

Strafella, L., \& Chapon, D. 2020, ArXiv e-prints [arXiv:2006.02759]

Sutherland, R. S., \& Dopita, M. A. 1993, ApJS, 88, 253

Tacchella, S., Dekel, A., Carollo, C. M., et al. 2016, MNRAS, 458, 242

Teyssier, R. 2002, A\&A, 385, 337

Tillson, H., Devriendt, J., Slyz, A., Miller, L., \& Pichon, C. 2015, MNRAS, 449, 4363

Tomassetti, M., Dekel, A., Mandelker, N., et al. 2016, MNRAS, 458, 4477

Tomczak, A. R., Quadri, R. F., Tran, K.-V. H., et al. 2014, ApJ, 783, 85

van der Wel, A., Franx, M., van Dokkum, P. G., et al. 2014, ApJ, 788, 28

van Dokkum, P. G., \& Brammer, G. 2010, ApJ, 718, L73

Wang, T., Schreiber, C., Elbaz, D., et al. 2019, Nature, 572, 211

Williams, C. C., Giavalisco, M., Cassata, P., et al. 2014, ApJ, 780, 1

Zolotov, A., Dekel, A., Mandelker, N., et al. 2015, MNRAS, 450, 2327 
S. Chabanier et al.: Compact galaxies in the Extreme-Horizon simulation

\section{Appendix A: Overview of the EH simulation}

Figure A.1 shows the large-scale structure of the EH simulation at redshift $z=2$. Figure A.2 displays the gas density in the CGM and IGM around a massive halo along with the spatial resolution achieved in the $\mathrm{EH}$ and $\mathrm{SH}$ simulation in the same region.

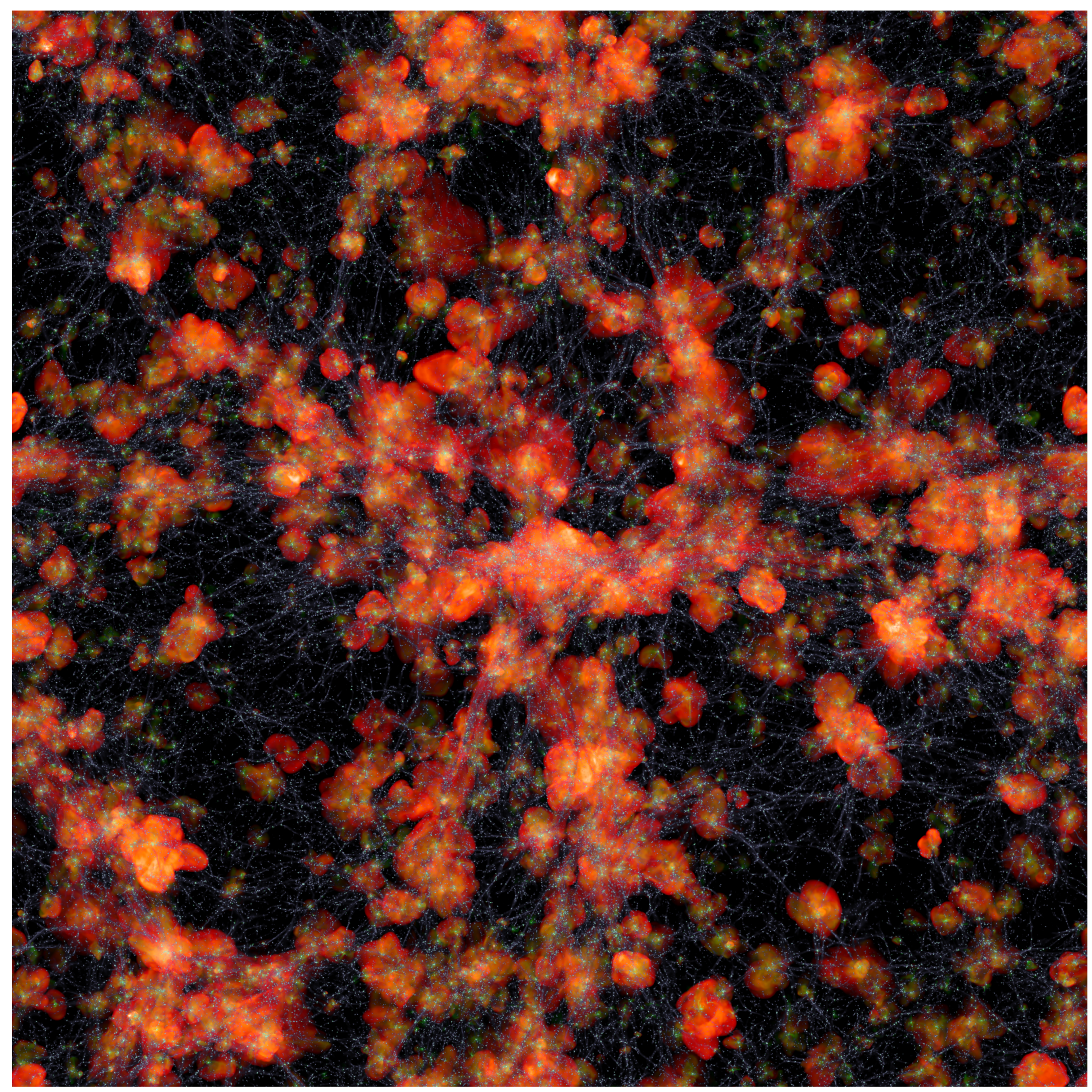

Fig. A.1. Projected map of the EH simulation at $z \simeq 2$. Gas density (grey), entropy (red), and metallicity (green) are shown. 

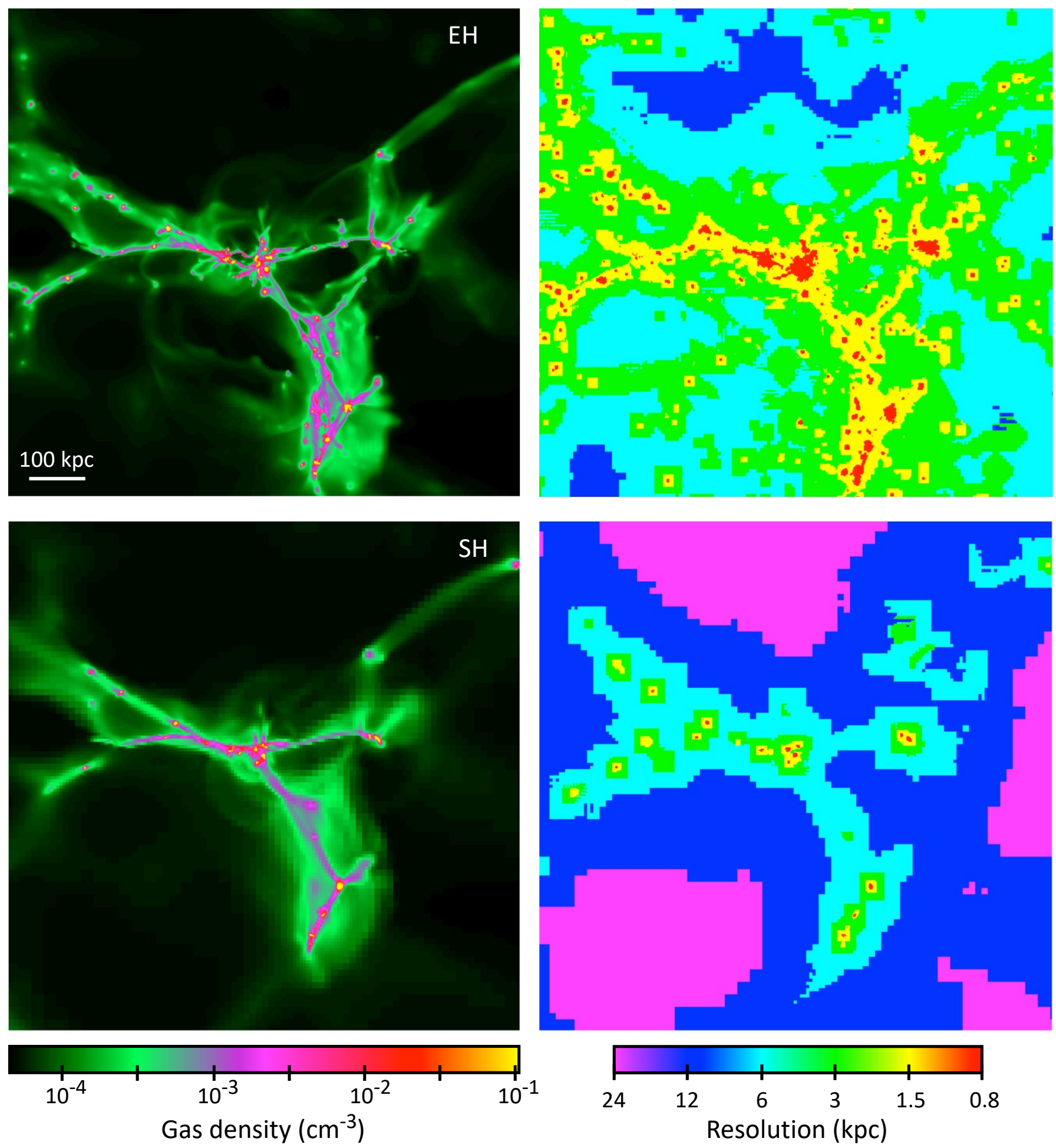

Fig. A.2. Projected density (left) and physical resolution (right) in EH (top) and SH (bottom) zoomed on a massive halo at $z=3$. The depth of the projections are $200 \mathrm{kpch}^{-1}$ and the boxes extend to $1 \mathrm{Mpch}^{-1}$ on each side. The gas density is computed as the mass-weighted average of local densities along the line of sight corresponding to each pixel. The resolution shown is the resolution of the cell in which the gas density is the highest along each line of sight. 


\section{Appendix B: Massive galaxies in $\mathrm{EH}$ and $\mathrm{SH}$}

Galaxy stellar mass maps from $\mathrm{EH}$ and $\mathrm{SH}$ are shown in Figs. B.1 and B.2, respectively. The slightly smaller sample size in $\mathrm{SH}$ compared to $\mathrm{EH}$ results from major mergers that do not occur at the very same time in both simulations and from a few galaxies that are just below the mass cutoff in $\mathrm{SH}$.

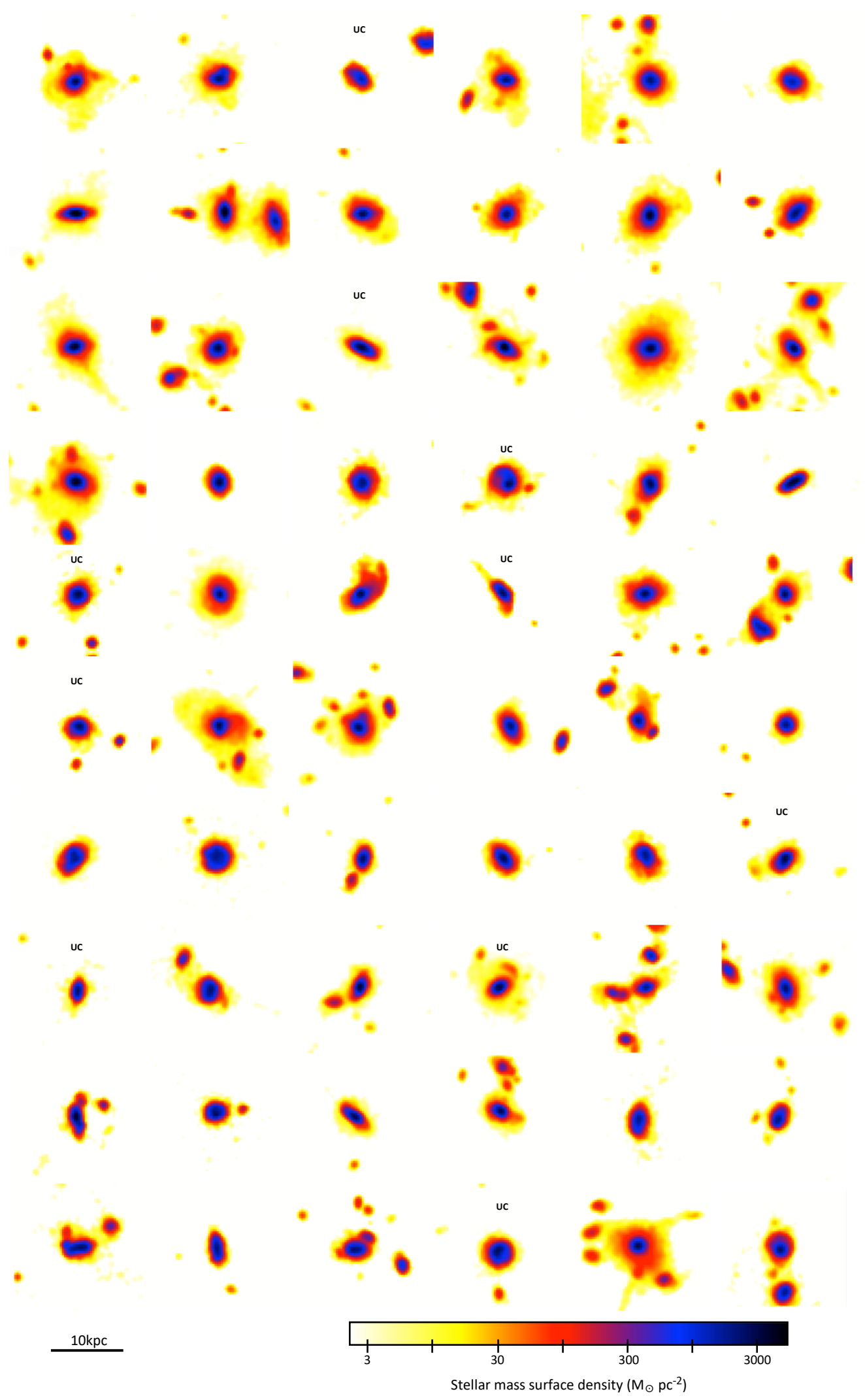

Fig. B.1. Stellar mass distribution of massive galaxies in the EH simulation. UC galaxies are flagged. 
A\&A 643, L8 (2020)

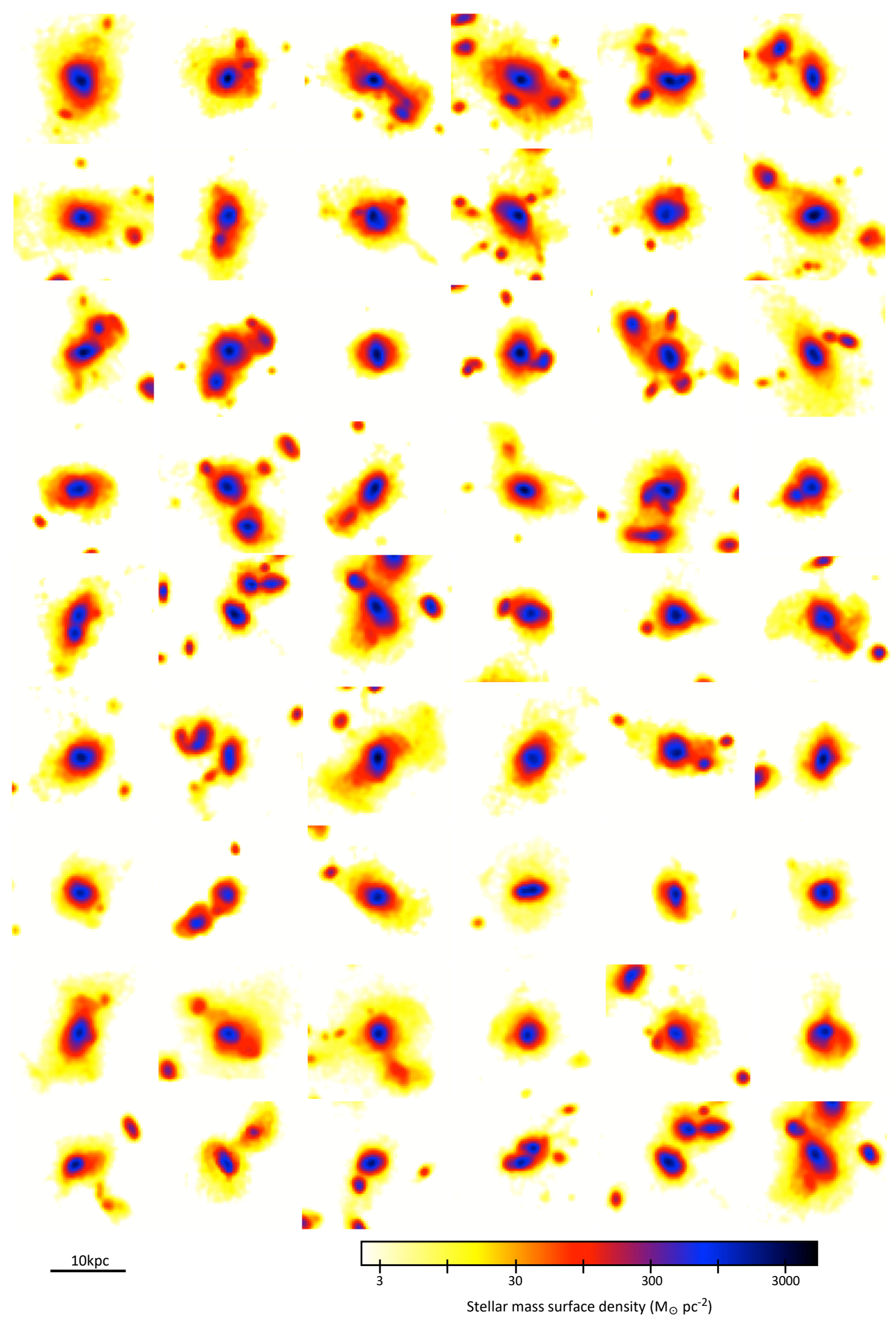

Fig. B.2. Stellar mass distribution of massive galaxies in the SH simulation. Galaxies are not meant to be individually matched to the SH galaxy given the independent samples were built in $\mathrm{EH}$ and $\mathrm{SH}$. 


\section{Appendix C: Environmental dependence}

To compare the environment of UC and NUC galaxies, we study the large-scale structure of the EH simulation with the persistent skeleton approach (Sousbie 2011) using the DISPERSE code (Sousbie 2013). The full skeleton is shown in Fig. C.1. Topological persistence can be used to characterise the significance of the structures depending on the local level of noise. Persistence levels from $3 \sigma$ to $8 \sigma$ are used to investigate different scales and prominences of the corresponding cosmic web (the larger the persistence threshold the lower the total number of filaments but those filaments are the most prominent ones). The $3 \sigma$ threshold ensures that we are not dominated by noise. On the other hand, above $8 \sigma$, very few structures remain. Figure C. 1 shows an intermediate situation with a persistence threshold at $7 \sigma$. In the remainder of this section, we will focus on two cases: (i) a low-persistence skeleton $(3 \sigma)$ where, by definition, all massive galaxies reside inside filaments and nodes which we can characterize (in terms for instance of density, connectivity, etc); and (ii) a high-persistence skeleton $(8 \sigma)$ which only selects the dominant filaments in the simulation box and for which we can measure the distance from galaxies to their closest filament or node.
At high persistence, the skeleton is sparse, dominated by a few dense and extended filaments. The UC and NUC galaxies both lie close to such filaments, as is expected for massive galaxies in general, but the galaxies that lie closest to these dense filaments and their nodes are never UC (Fig. C.2, top panel). Instead, UC galaxies tend to lie in intermediate-density filaments, as shown in the analysis of the closest filaments in a lower-persistence skeleton analysis (Fig. C.2, bottom panel). This is consistent with the previous results on the merger history of UC galaxies, as objects in the densest regions of the main filaments are expected to form their main progenitor early-on and subsequently grow by minor mergers or diffuse accretion, or a combination of both. Nevertheless, the UC galaxies still do form in dense regions and none are found in low-density filaments, where smooth accretion would dominate over mergers (Fig. C.2 bottom panel and Fig. C.1 for a visualization).

Hence, UC galaxies are expected to be found in relatively dense environments, but not in the very densest filaments and nodes. Galaxies in the densest regions of the cosmic web are expected to be rarely ultra-compact at $z \sim 2$, yet they could undergo ultra-compact phases at higher redshift if their early formation involves major mergers of numerous low-mass progenitors.

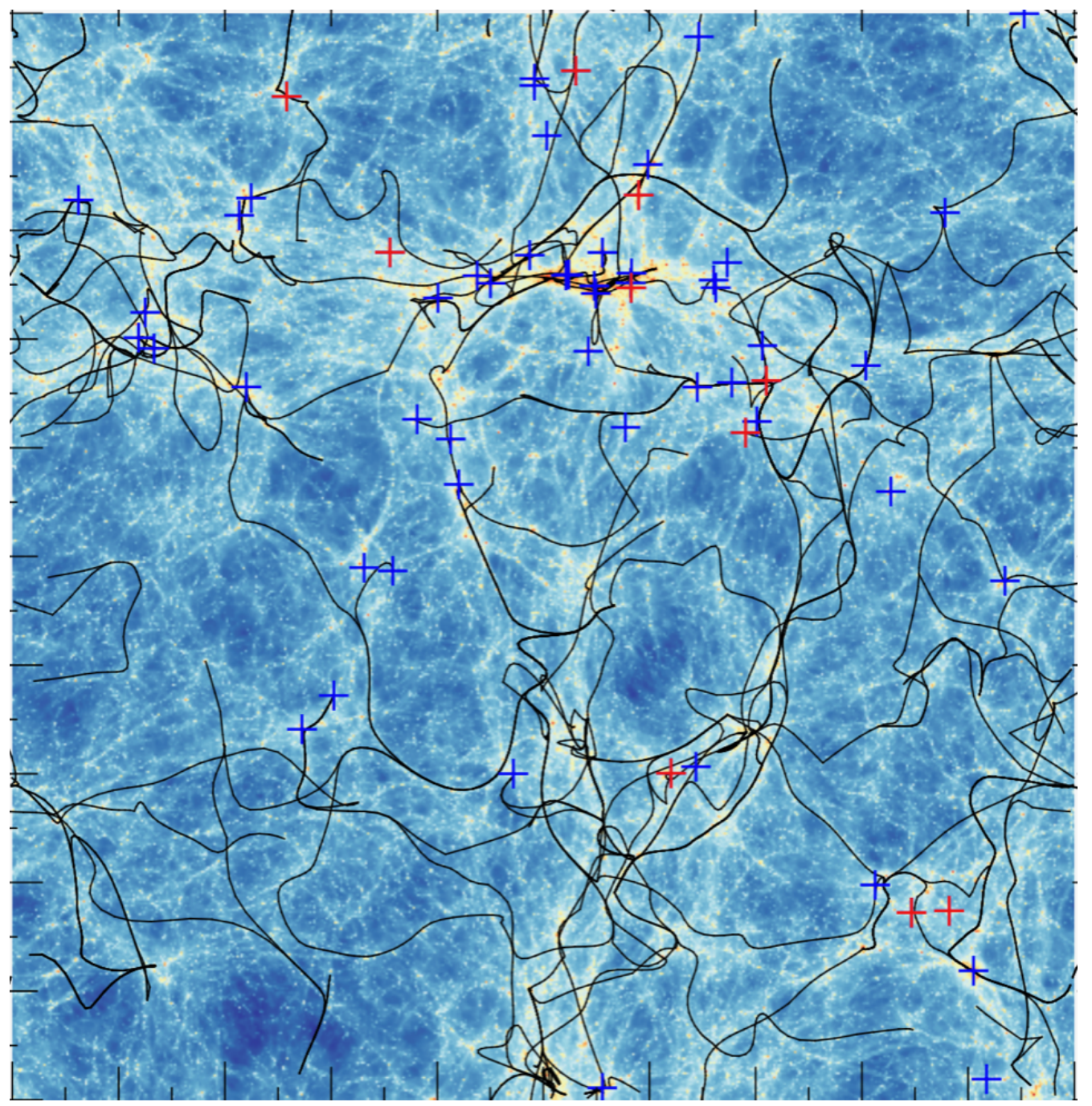

Fig. C.1. Projected EH skeleton of the $50 \mathrm{Mpch}^{-1}$ box with a $7 \sigma$ persistence level at $z=2$. Crosses indicate the projected position of massive galaxies, with red crosses for UC and blue crosses for NUC. 
A\&A 643, L8 (2020)

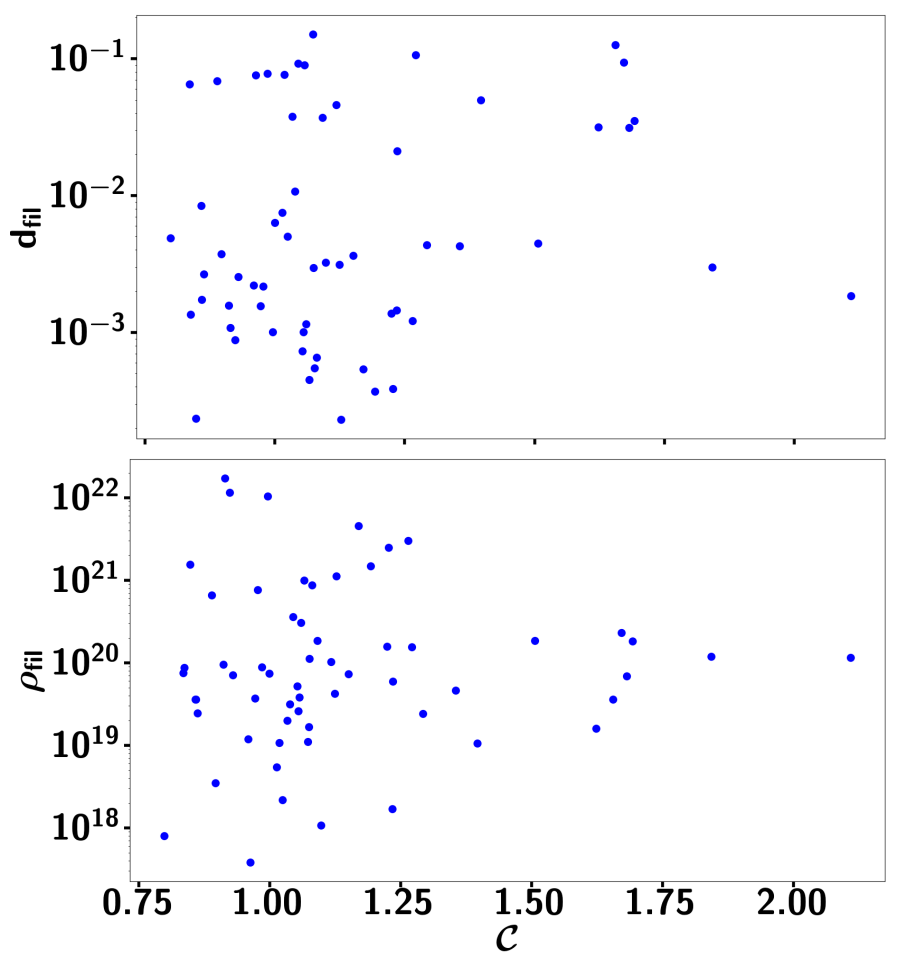

Fig. C.2. Top panel: distance to the closest filament $d_{\text {fil }}$ (in box size units) of the $8 \sigma$ sparse skeleton for all EH massive galaxies as a function of their compactness. Bottom panel: density in the closest filament $\rho_{\text {fil }}$ (obtained by DTFE from a mass-weighted Delaunay tessellation of the galaxy catalogue) of the $3 \sigma$ dense skeleton for all $\mathrm{EH}$ massive galaxies as a function of their compactness. For UC galaxies, exclusion zones are clearly visible at small distance to the filaments and in the very low and very high density regions, as compared to the NUC. 